\title{
Numerical approach to low-doping regime of the $\mathrm{t}-\mathrm{J}$ model
}

\author{
J. Bonča, ${ }^{1,2}$ S. Maekawa, ${ }^{3,4}$ and T. Tohyama ${ }^{5}$ \\ ${ }^{I}$ Faculty of Mathematics and Physics, University of Ljubljana, Ljubljana, Slovenia \\ ${ }^{2}$ J. Stefan Institute, Ljubljana, Slovenia \\ ${ }^{3}$ Institute for Materials Research, Tohoku University, Sendai 980-8577, Japan \\ ${ }^{4}$ CREST, Japan Science and Technology Agency (JST), Kawaguchi, Saitama 332-0012, Japan \\ ${ }^{5}$ Yukawa Institute for Theoretical Physics, Kyoto University, Kyoto 606-8502, Japan
}

(Dated: September 15, 2021)

\begin{abstract}
We develop an efficient numerical method for the description of a single-hole motion in the antiferromagnetic background. The method is free of finite-size effects and allows calculation of physical properties at an arbitrary wavevector. Methodical increase of the functional space leads to results that are valid in the thermodynamic limit. We found good agreement with cumulant expansion, exact- diagonalization approaches on finite lattices as well as self-consistent Born approximations. The method allows a straightforward addition of other inelastic degrees of freedom, such as lattice effects. Our results confirm the existence of a finite quasiparticle weight near the band minimum for a single hole and the existence of string-like peaks in the single-hole spectral function.
\end{abstract}

PACS numbers: 71.10.Fd,71.10.Pm,74.25.Jb,79.60.-i

\section{INTRODUCTION}

A description of hole motion in the antiferromagnetic (AFM) background as described by the $t-J$ model represents one of the long-standing, open, theoretical problems in the field of correlated systems. The accurate solution of this problem may be crucial for understanding the behavior of high-temperature superconductors in the underdoped regime. Apart from the analytical solution in the Nagaoka regime [1] in the limit of zero doping and small $J / t$, as well as rigorous theorems in the symmetric point $J=2 t$ [2], there are no exact solutions of this model in two spacial dimensions. Many outstanding, early approaches to this problem, such as the self-consistent Born approximation (SCBA) [3, 4, 5, 6, 7], self-consistent perturbational approach (SCPA) [6], calculations based on the string picture [8, 9, 10], cumulant expansion (CE) technique [11], exact diagonalization (ED) calculations on small clusters [12, 13, 14], quantum Monte carlo calculations [15], and recent state-of-the-art QMC calculations [16, 17, 18] have provided quantitative description of the quasiparticle band-width, effective mass, and quasiparticle weight. Most of these methods reproduce dynamical properties, such as the one-hole spectral function, as well.

Among these approaches, ED calculations on small clusters provide exact solutions of the $t-J$ Hamiltonian but suffer from finite-size effects due to small system sizes. Similarly, QMC calculations are limited to small, even though larger clusters. In addition, analytic continuation is necessary to obtain spectral properties, since most of QMC methods compute Green's function, defined in imaginary time. The SCBA and SCPA calculations are likewise limited to finite-size calculations in momentum space. Furthermore, they seem to overemphasize the string effect. On the other hand, early calculations based on the string picture are similar to the concept of the linear combination of atomic orbitals, which provide results for arbitrary momentum transfer. However, previous results $[9,10,19]$ are not necessarily comparable with SCBA, SCPA and ED results of the $t-J$ model. This is predominantly due to a limited number of variational parameters or to the small size of the Hilbert space used in these calculations. In comparison to the variational approach used in Ref. [19], where the authors use a similar method for construction of the functional basis set, our method employs an exact-diagonalization approach using Lanczos technique, which allows solutions of much larger Hilbert spaces.

The aim of this work is to present an accurate exact diagonalization method, defined over a limited functional space (EDLFS). The method is based on the string picture [8, 9, 10], which provides solutions to a single-hole problem in the AFM background that are free of finite-size effects. Furthermore, the method takes the advantage of modern computing capabilities that allow solutions of large matrices. Through the efficient construction of the limited functional space (LFS), even when using only a few thousand states, this method provides results that can be directly compared to the state-of-the-art numerical approaches on small lattices [14] that require tens of millions of states.

Despite much work in this area [20] there remain many open questions concerning the physics of a doped AFM in the zero-doping limit. Current interest in this field is in part focused on the influence of the electron-phonon interaction on correlated motion of a hole in the AFM background [17, 21, 22, 23]. Another open question concerns the proper description of the difference between the hole- and electrondoped cuprates [24]. There is also a need for a method that would resolve the issue of a disappearing quasiparticle weight that was predicted in a thermodynamic limit of a doped AFM due to the phase string effect [25]. 


\section{METHOD}

We start by writing the $t-J$ model as

$$
\begin{aligned}
H & =H_{t}+H_{\|}+H_{\perp} \\
H_{t} & =-t \sum_{\langle\mathbf{i}, \mathbf{j}\rangle, s} \tilde{c}_{\mathbf{i}, s}^{\dagger} \tilde{c}_{\mathbf{j}, s}+\text { h.c. } \\
H_{\|} & =J \sum_{\langle\mathbf{i}, \mathbf{j}\rangle} S_{\mathbf{i}}^{z} S_{\mathbf{j}}^{z} \\
H_{\perp} & =J / 2 \sum_{\langle\mathbf{i}, \mathbf{j}\rangle} S_{\mathbf{i}}^{+} S_{\mathbf{j}}^{-}+S_{\mathbf{i}}^{-} S_{\mathbf{j}}^{+},
\end{aligned}
$$

where $\tilde{c}_{\mathbf{i}, s}=c_{\mathbf{i}, s}\left(1-n_{\mathbf{i},-s}\right)$ is a fermion operator, projected onto a space of no double occupancy. The sums run over the pairs of nearest neighbors as well as over the two spin orientations. Following in part works by Trugman [9], Inoue and Maekawa [19], and El Shawish and J.B. [26], we construct the LFS starting from a Néel state with one hole, $\left|\phi_{\mathbf{k}}^{(0)}\right\rangle=c_{\mathbf{k}} \mid$ Neel $\rangle$ and proceed with generation of new states, by application of the kinetic part of the Hamiltonian $H_{t}$, i.e.

$$
\left\{\left|\phi_{\mathbf{k} l}^{\left(N_{h}\right)}\right\rangle\right\}=H_{t}^{N_{h}}\left|\phi_{\mathbf{k}}^{(0)}\right\rangle .
$$

This procedure generates strings with maximum lengths given by $N_{h}$. While constructing the LFS, translation symmetry, generated by two minimal translations $\mathbf{r}_{1,2}=(1, \pm 1)$, is taken into account. Due to exponentially rapid growth of the LFS with increasing $N_{h}$ we introduce an additional parameter $N_{b} \leq N_{h}$ that restricts generation of long strings by imposing a condition under which all coordinates of spin-flips should satisfy $\left|\mu_{h}-\mu_{f}\right| \leq N_{b} ; \mu=\{x, y\}$ where $h$ and $f$ refer to electron and spin-flip indexes, respectively. Application of this condition improves the quality of the LFS by increasing the number of states containing spin-flips in the vicinity of the hole while keeping the total amount of states within computationally accessible limits. The full Hamiltonian in Eq. 2 is then diagonalized within this LFS using the standard Lanczos procedure. We also note that our method, even though defined on the infinite lattice, is variational. Increasing the number of LFS systematically lowers energies of the zero- and singlehole states, i.e. $E^{0 h}$ and $E^{1 h}$.

While generation of single-hole states through application of only the kinetic part of the Hamiltonian seems a rather natural choice for the construction of the single-hole wavefunction, there remains a question of how to construct the LFS for the undoped case, i.e., the Heisenberg model. The solution of the latter seems necessary in order to compute spectral properties of the one-hole system as well as its energy, relative to the undoped case. We next assume that the spacial extent of the disturbance of the spin background around the doped hole (in the literature also referred as a magnetic polaron) is finite. In this case it is not necessary to obtain the exact solution of the undoped system on the infinite 2D lattice for the correct description of the single hole properties. It is sufficient to find a solution of the Heisenberg model in the vicinity of the doped hole. We therefore construct the 0-hole LFS using the 1-hole LFS by simply filling the empty space with a spin.

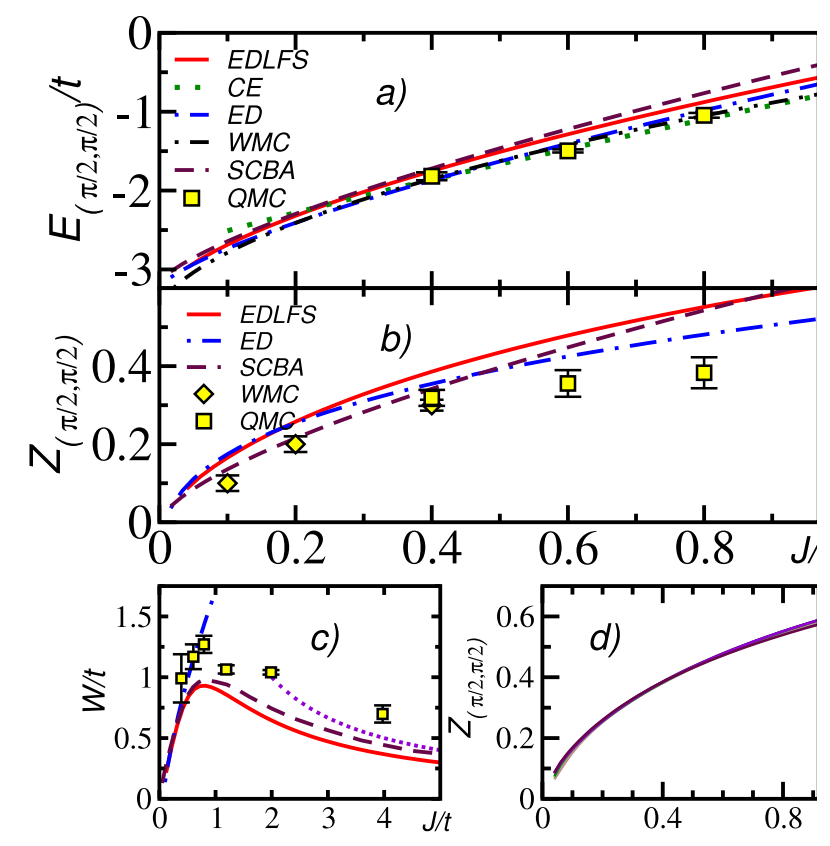

Figure 1: (Color online) a) Single-hole energy $E_{\mathbf{k}=(\pi / 2, \pi / 2)}$ vs. $J / t$. The full line represents EDLFS results of the present work that were obtained using $N_{s t}=37402972$ states. Dotted line represent CE results from Ref. [11]. The dot-dashed line, given by $E_{\mathbf{k}=(\pi / 2, \pi / 2)}=$ $J / t-3.24+2.65(J / t)^{0.72}$ is an interpolation based on ED results obtained on 32 sites [14]. The double-dot-dashed line represents interpolation $E_{\mathbf{k}=(\pi / 2 . \pi / 2)}=-3.36+3.50(J / t)^{2 / 3}$ based on WMC method from Ref. [16]. The dashed line, $E_{\mathbf{k}=(\pi / \mathbf{2}, \pi / \mathbf{2})}=$ $-3.17+2.83(J / t)^{0.73}$ is a result of SCBA calculation, [5, 27]. Squares represent QMC calculations from Ref. [18]. Note that $E_{\mathbf{k}} / t$ results of $\mathrm{ED}, \mathrm{WMC}$ and $\mathrm{QMC}$ calculations were shifted by $-J / t$ due to a different deffinition of the $t-J$ model. b) Quasiparticle weight $Z_{\mathbf{k}=(\pi / 2, \pi / 2)}$ vs. $J / t$. The full line represents EDLFS calculation using $N_{s t}=37402972$ states. The dot-dashed line represents ED results as summarized in the extrapolation given by $Z_{\mathbf{k}=(\pi / 2, \pi / 2)}=-0.136+0.664(\mathrm{~J} / t)^{0.333}$ [14], while the dashed line represents SCBA calculation $Z_{\mathbf{k}=(\pi / 2, \pi / 2)}=0.63(\mathrm{~J} / t)^{0.667}$ from Ref. [5] (similar extrapolation was also obtained using SCPA in Ref. [6]). Diamonds represent WMC calculation from Ref. [16], and squares are QMC results from Ref. [18]. Note that WMC and QMC data were multiplied by a factor of 2 due to a different definition of $Z_{\mathbf{k}}$ used in Refs. [16, 18]. c) The bandwidth $W / t$ vs. $J / t$ calculated with EDLFS (full line), ED [14] (dot-dashed line), SCBA [5](dashed lines), QMC calculations from Ref. [18] (squares), and $W / t=2 t^{2} / J$ (dotted line), proposed in Ref. [5]. d) $Z_{\mathbf{k}}$ vs. $J / t$ (ten nearly overlapping curves) obtained by using 10 different sizes of LFS as explained in the text.

\section{STATIC PROPERTIES}

We now turn to the numerical results. The one-hole energy, measured from the energy of the undoped system, $E_{\mathbf{k}}=$ $E_{\mathbf{k}}^{1 h}-E^{0 h}$, is shown at the one-hole band-minimum $\mathbf{k}=$ 


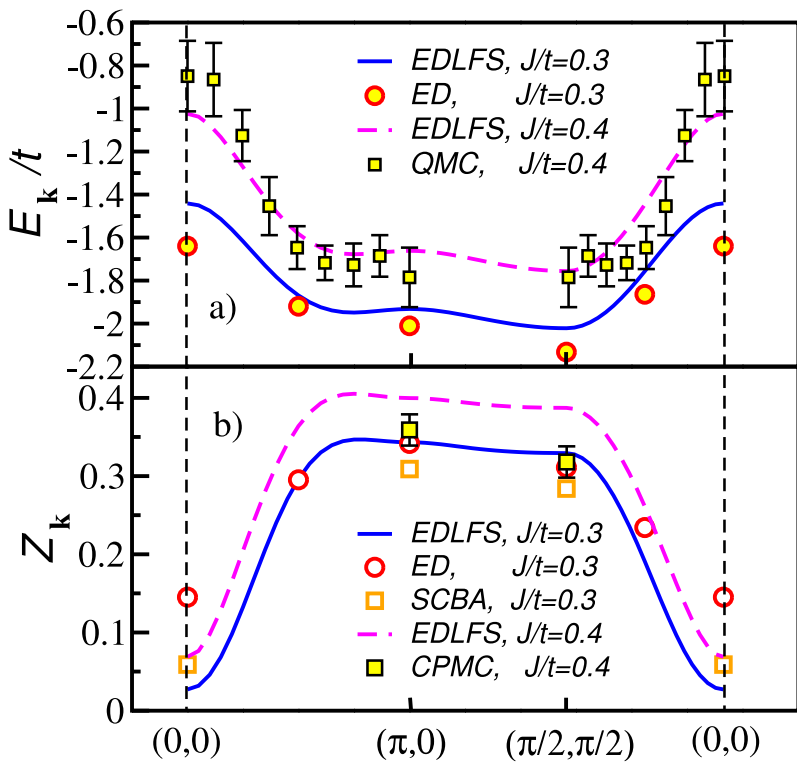

Figure 2: (Color online) a) Single-hole energy $E_{\mathbf{k}}$ and b) quasiparticle weight $Z_{\mathbf{k}}$. Both quantities were calculated at fixed $J / t=0.3$ and 0.4 as noted in the figure. EDLFS results of the present work were obtained with $N_{s t}=37402972$ states (full lines), ED results (circles), calculated on a 32 site system, are from Ref. [14], QMC results (squares) are from Ref. [18], while SCBA results (diamonds) in b) are from Ref. [5].

$(\pi / 2, \pi / 2)$ in Fig. 11, along with CE [11], ED [14], worm quantum Monte Carlo (WMC) [16, 17], Quantum Monte Carlo (QMC) calculations [18], and SCBA [5, 27] results. While our method is defined on the infinite system, the absolute values of $E_{\mathbf{k}}^{1 h}$ and $E^{0 h}$ are ill-defined since they grow with the increasing number of basis states as the number of spin-flips, generated by the hole motion, increases. In contrast, $E_{\mathbf{k}}$ remains finite and well defined. Although our method can not be directly compared to the cumulant expansion technique [11], we use some of the aspects of this technique. Since we use in our method only the hopping part of the Hamiltonian, Eq. 22 to generate new states, all spin-flips are by construction limited to the vicinity of the hole. This by no means restricts the LFS only to connected strings. A propagating hole can also generate disconnected strings. In our approach, the precision of the description of the quantum spin fluctuations, caused by the presence of the hole, increases with decreasing distance from the hole. We can therefore expect to achieve a thermodynamic limit as soon as the extent of the spin-flips in the LFS exceeds the size of the magnetic polaron. In addition, we should stress that the zero-hole energy, $E^{0 h}$, per se has no physical meaning. It simply represents the solution of the Heisenberg model, defined on the zero-hole LFS, that is identical to the one-hole LFS with the exception of the additional spin located on the hole position. The high efficiency of our approach is reflected in good agreement of our results with CE method, [11], and SCBA approach [5, 27]. For comparison we also present results obtained with ED calculation on a 32 site system [14], WMC calculations [16], as well as with QMC calculations [18] performed on much larger lattices (24x24) (see Fig.11). In general, EDLFS, CE as well as SCBA methods give consistently lower values of the singlehole (polaron) energy in comparison to ED and QMC methods. Here we stress, that the single-hole energy is extremely sensitive to the appropriate choice of the LFS for the 1- as well as of the 0- hole space. Our results can be almost perfectly fitted with a form $E_{(\pi / 2, \pi, 2)} / t=\omega_{0}=a_{0}+b_{0}(J / t)^{\gamma_{0}}$ where parameters $a, b$ and $\mathbf{k}=0$ are listed in the first row of Table

We present the bandwidth $W$ in Fig. 1te along with SCBA [5] and QMC results [18], as well as with analytical prediction $W=2 t^{2} / J$ [5], valid in the large $J / t$ limit. We find good agreement with ED results in the physically most relevant regime $J / t \sim 0.4$. We note that in Ref. [14] $W$ is defined as $W=E_{(\pi, \pi)}-E_{(\pi / 2, \pi / 2)}$. In our approach due to broken translation symmetry the point $\mathbf{k}=(\pi, \pi)$ is folded onto the $\mathbf{k}=0$ point. We thus believe that our definition of $W$ is comparable to the one in Ref. [14]. QMC results from Ref. [18] in contrast to EDLFS, ED and SCBA results predict slightly larger values of $W / t$. Note however larger error bars in QMC results around $J / t \lesssim 0.6$.

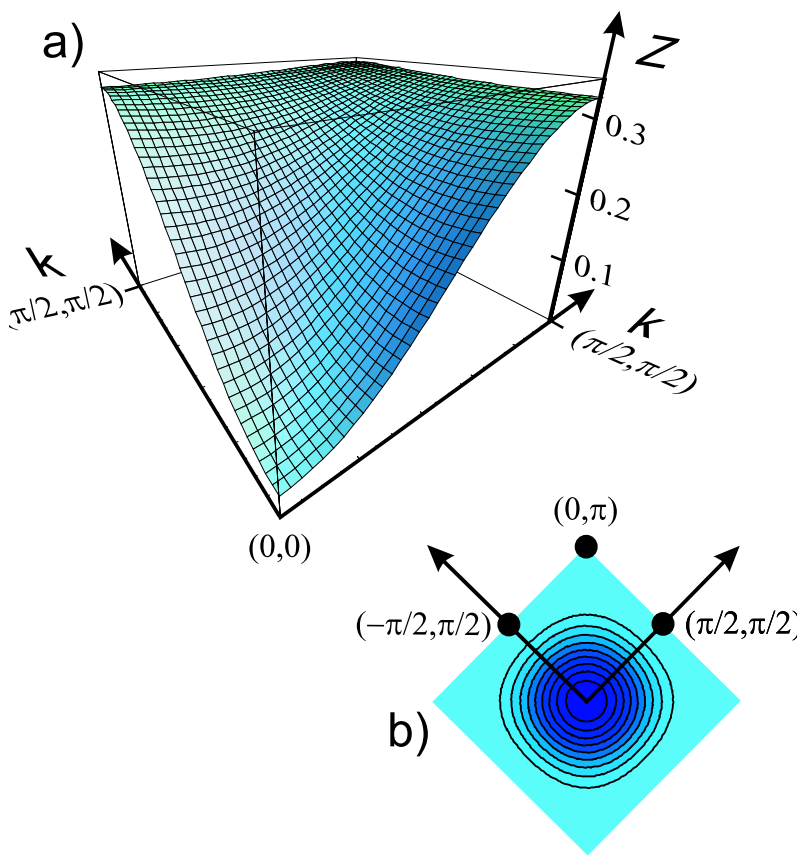

Figure 3: (Color online) a) Surface plot of $Z_{\mathbf{k}}$ at $J / t=0.3$, computed on a mesh of $400 \mathbf{k}-$ points. Variational space with $N_{s t}=5213618$ was used. Only $1 / 4$ of the AFM BZ is shown. b) Contour plot of $Z_{\mathbf{k}}$ with equidistant contours while wavevectors are spanning the whole AFM BZ. The rest is the same as in a).

Our calculation of $E_{\mathbf{k}}$ presented in Fig. $2 \mathrm{k}$ reflects another important advantage of the present method over ED calculations on limited system sizes. Note, however, that our calculations are limited to the reduced AFM Brillouin zone (BZ) because of broken translational symmetry. Defining the LFS on an infinite lattice allows calculation of physical properties at an arbitrary wavevector, limited to AFM BZ. In Fig. 2a, we 
present the dispersion relation $E_{\mathbf{k}}$, calculated at $J / t=0.3$ and 0.4. Taking into account the fact that we are computing $E_{\mathbf{k}}$ in absolute units (we used no additional energy shift), we find good agreement with ED results [14], in particular when comparing the $\mathbf{k}$-dependence of the single-hole energy and the bandwidth. We find qualitative agreement also with QMC results from Ref. [18], calculated at $J / t=0.4$. QMC method predicts larger bandwidth as also seen in Fig. 1c. To further quantify the efficiency of our method, we present our results for $E_{\mathbf{k}}$ at selected $\mathbf{k}$ - points in Table $\amalg$ These results were obtained using different numbers of states spanning the LFS at the physically relevant value $J / t=0.3$. It is encouraging that reasonable results for the one-hole energy can be obtained from a LFS as small as $N_{s t}=1121$.

So far, we have shown that our method is successful in obtaining the ground-state energy of the magnetic polaron, however, the current scientific interest and existing open problems primarily concern dynamic properties of a doped hole. Before moving to dynamic response, we next present our results of a closely related quantity, i.e. the quasiparticle weight, $Z_{\mathbf{k}}$, vs. $J / t$, see Fig. 1b. We define $Z_{\mathbf{k}}$ by

$$
Z_{\mathbf{k}}=\frac{\left|\left\langle\Psi_{\mathbf{k}, 0}^{1 h}\left|c_{\mathbf{k}}\right| \Psi^{0 h}\right\rangle\right|^{2}}{\left\langle\Psi^{0 h}\left|c_{\mathbf{k}}^{\dagger} c_{\mathbf{k}}\right| \Psi^{0 h}\right\rangle}
$$

where $\Psi_{\mathbf{k}, 0}^{1 h}\left(\Psi^{0 h}\right)$ represent the ground state of the system with either one or zero holes. Note that the ground state $\Psi^{0 h}$ has $\mathbf{k}=0$. The agreement with the ED calculation is surprisingly good for $J / t \lesssim 0.3$. The WMC calculation from Ref. [16] and QMC calculations from Ref. [18] yield slightly smaller values for $Z_{\mathrm{k}}$. It is noteworthy mentioning that the two different QMC methods yield consistent values of $Z_{\mathbf{k}}$ (note nearly perfect overlap between the two methods at $J / t=0.4)$. We have also tested the $J / t \rightarrow \infty$ limit where exact result based on the spin-wave approximation from Ref. [28] yields $Z=0.822$. Our method gives $Z=0.926$, which can be further compared with SCBA result calculation that gives $Z=1$.

We next briefly discuss possible sources of errors affecting results obtained by different approaches. In case of SCBA calculations, the error is due to the approximate nature of the calculation since only non-crossing diagrams are taken into account. ED calculations are limited to small latticesizes that may lead biased results due to finite-size effects. QMC simulation from Ref. [18], based on the loop-cluster Monte carlo method for the AFM state and the hole propagation within the fixed spin background, yields increasing larger error bars as one approaches the physically relevant regime $J / t \lesssim 0.6$, while WMC method [16] suffers from he minus-sign problem. EDLFS naturally depends on the choice of the LFS. Increasing the number of LFS should yield results that are free of finite-size effects and valid in the thermodynamic limit. Nevertheless, a systematic error may occur due to a particular algorithm used to create different LFS, Eq. 2. To demonstrate the stability of our results against the choice of different LFS, as well as a rapid convergence of

\begin{tabular}{|l|ccc|}
\hline & $a_{n}$ & $b_{n}$ & $\gamma_{n}$ \\
\hline$\omega_{0}$ & -3.37 & 2.86 & 0.62 \\
$\omega_{1}$ & -3.39 & 4.50 & 0.76 \\
$\omega_{2}$ & -3.12 & 5.56 & 0.72 \\
\hline
\end{tabular}

Table I: Fitting parameters of the lowest peak positions in $A_{(\pi / 2, \pi / 2)}(\omega)$. Fits have the from $\omega_{n}=a_{n}+b_{n}(J / t)^{\gamma_{n}}$.

our method for $0.02 \lesssim J / t<1$ with increasing $N_{s t}$, we present in Fig. (1d) nine nearly overlapping curves depicting $Z_{\mathbf{k}}$. The curves were calculated using different LFS's with: $N_{s t}=7610,9786,43884,80108,218950,642406,912478$, 3109626 and 5213618 as obtained using LFS generator, Eq. 2 with various values of $N_{h}$ and $N_{b}$. The close agreement of values for $Z_{\mathbf{k}}$ given in Table $\amalg$ represents additional qualitative demonstration of convergence in our calculation. Note that results are only weakly dependent upon the choice of parameters $N_{h}$ and $N_{b}$ that define the generating algorithm for LFS.

In Fig 2b we present $Z_{\mathbf{k}}$ along the special symmetry lines in the reduced AFM BZ. The agreement with the ED result is good for large values of $k=|\mathbf{k}|$ while the agreement with SCBA calculation is poorer. The discrepancy between our method and SCBA is similar over the whole AFM BZ since the SCBA does not suffer from finite-size effects. Most importantly, we find the value of $Z_{\mathbf{k}}$ to be very small around the $\mathbf{k}=0$ point (see also Table I), followed by a sharp increase with increasing $k$. These observations are consistent with the SCBA result.

A surface plot of $Z_{\mathbf{k}}$ in Fig. 3a that consist of $400 k$-points, calculated on a system with $N_{s t}=5213618$ states, shows the power of our method. As expected from results, plotted in Fig. 2, $Z_{\mathbf{k}}$ shows a pronounced minimum located at $\mathbf{k}=0$ followed by a rapid increase with increasing distance from the $\mathbf{k}=0$ point. In Fig. 3b we show contour plot of $Z_{\mathbf{k}}$ over the whole AFM BZ where contour lines, representing values of $Z_{\mathbf{k}}$, are uniformly spaced in the interval $[0,0.3]$.

\section{SPECTRAL FUNCTIONS}

In Figs. 4h-c we plot the hole spectral function $A_{\mathbf{k}}(\omega)$, calculated at $J / t=0.3$ using three typical values of $\mathbf{k}$. We define $A_{\mathrm{k}}(\omega)$ as

$$
A_{\mathbf{k}}(\omega)=\sum_{n}\left|\left\langle\Psi_{\mathbf{k}, n}^{1 h}\left|c_{\mathbf{k}}\right| \Psi^{0 h}\right\rangle\right|^{2} \delta\left(\omega-\left(E_{\mathbf{k}, n}^{1 h}-E^{0 h}\right)\right),
$$

where $\left|\Psi_{\mathbf{k}, n}^{1 h}\right\rangle$ and $E_{\mathbf{k}, n}^{1 h}$ represent excited states and energies of the 1-hole system. In many respects, our results agree with the ED calculations of Leung and Gooding, Ref. [14]. The quasiparticle peak is well defined for wavevectors lying on the edge of the AFM BZ. In particular, for $\mathbf{k}_{1}=(\pi / 2, \pi / 2)$ the 


\begin{tabular}{|c|c|c||ccc||ccc|}
\hline$N_{h}$ & $N_{b}$ & $N_{s t}$ & \multicolumn{3}{|c||}{$E_{\mathbf{k}}$} & \multicolumn{3}{|c}{$Z_{\mathbf{k}}$} \\
\hline \hline 6 & 4 & 1121 & -2.01925 & -1.95213 & -1.44065 & 0.29253 & 0.32780 & 0.00002 \\
8 & 4 & 7610 & -1.99475 & -1.92799 & -1.47960 & 0.32617 & 0.33895 & 0.03093 \\
8 & 8 & 9786 & -1.99951 & -1.92888 & -1.47982 & 0.32349 & 0.33803 & 0.03077 \\
10 & 4 & 43884 & -1.98751 & -1.92209 & -1.45354 & 0.32529 & 0.35097 & 0.03359 \\
10 & 8 & 80108 & -2.00182 & -1.92305 & -1.45542 & 0.32486 & 0.34104 & 0.03098 \\
12 & 4 & 218950 & -2.00272 & -1.93757 & -1.46192 & 0.32790 & 0.34895 & 0.03535 \\
12 & 8 & 642406 & -2.01059 & -1.92709 & -1.43991 & 0.32633 & 0.34345 & 0.03009 \\
14 & 4 & 912478 & -2.00024 & -1.93322 & -1.47915 & 0.32902 & 0.34942 & 0.03907 \\
14 & 8 & 4992876 & -2.01830 & -1.93175 & -1.44255 & 0.32805 & 0.34314 & 0.02809 \\
14 & 12 & 5225818 & -2.01831 & -1.93175 & -1.44255 & 0.32804 & 0.34314 & 0.02809 \\
16 & 8 & 37402972 & -2.02175 & -1.93205 & -1.44112 & 0.32939 & 0.34324 & 0.02713 \\
\hline
\end{tabular}

Table II: $E_{\mathbf{k}}$ and $Z_{\mathbf{k}}$, calculated for $J / t=0.3$ and different sizes of the LFS, generated using different values of $N_{h}$ and $N_{b}$.

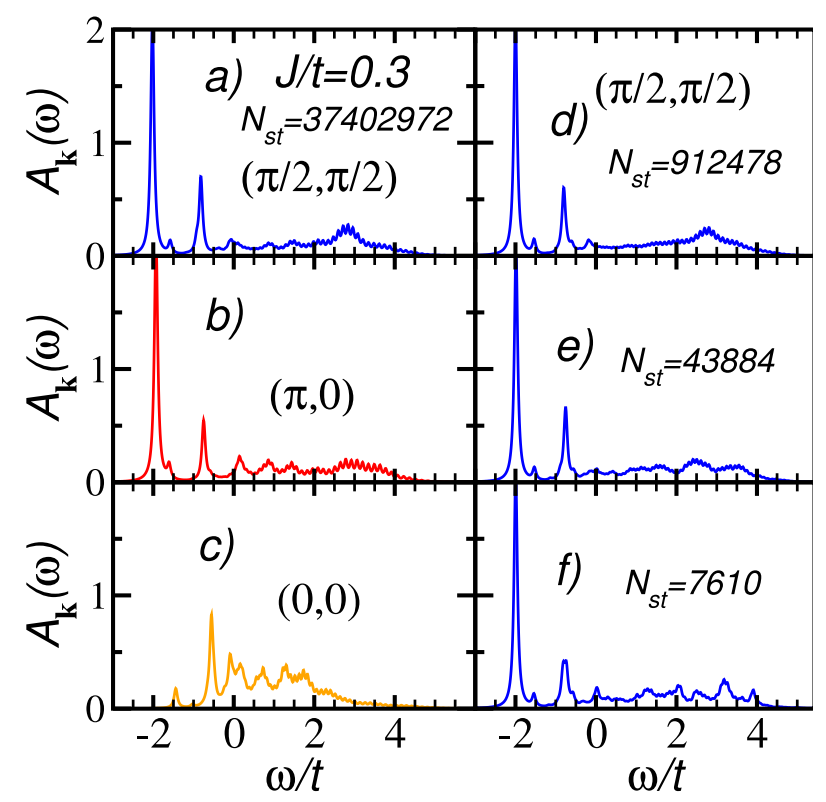

Figure 4: (Color online) a-c) Spectral functions $A_{\mathbf{k}}(\omega)$ for three typical values of $\mathbf{k}$, d-f) $A_{\mathbf{k}}(\omega)$ at $\mathbf{k}=(\pi / 2, \pi / 2)$ calculated using different sizes of LFS's as indicated in the insets. In all cases, we have used $J / t=0.3$ and an artificial damping of $\epsilon=0.05$.

peak is located at $\omega=\omega_{0}=E_{\mathbf{k}_{1}}$, see also Table【 The quasiparticle peak is well defined also for $\mathbf{k}_{2}=(\pi, 0)$. In contrast to ED results, we see a tiny peak at $\mathbf{k}_{1}$, located at $\omega_{1} \sim-1.58$ that scales with $J / t$ as $\omega_{1}=a_{1}+b_{1}(J / t)^{\gamma_{1}}$, Table 【 This fit is valid in the regime $0.3 \lesssim J / t \lesssim 1.0$. This peak can also be distinguished at $\mathbf{k}_{2}$. At yet higher frequencies there is another well defined peak. It is located at $\omega_{2} \sim-0.81$ at $\mathbf{k}_{1}$ with the following scaling $\omega_{2}=a_{2}+b_{2}(J / t)^{\gamma_{2}}$, Table【. This fit is valid in the regime $0.1 \lesssim J / t \lesssim 1.0$. ED results from Ref. [14] also display a well defined but less sharp structure at these frequencies. Moving towards $\mathbf{k}_{2}$, this peak looses some weight, however, it remains well defined. Spectrum at larger $\omega$ is broad and mostly featureless. We note different scaling with $J / t$ between the quasiparticle peak, located at $\omega_{0}$ and string-like peaks, positioned at $\omega_{1}$ and $\omega_{2}$, see Table 1 .

At $\mathbf{k}_{3}=0, A_{\mathbf{k}_{3}}(\omega)$ displays a much smaller quasiparticle peak at $\omega \sim E_{\mathbf{k}_{3}}$ than found in ED calculations. The broad, mostly incoherent part moves to lower frequencies and slightly shrinks. Nevertheless, the incoherent structure is broader than in ED calculations.

In Figs 4h,d-e we plot $A_{\mathbf{k}_{1}}(\omega)$ for 4 different sizes of the LFS, ranging from $N_{s t}=37402972$ down to 7610 . A filling up of the incoherent part of the spectrum in the large $\omega$ interval $0 \lesssim \omega / t \lesssim 4$ is the predominant effect of increasing $N_{s t}$. All special features in the range $\omega \lesssim 0$ seem to be well captured within the smallest size LFS. In addition, spectral functions for the largest two LFS's (see Figs $4 \mathrm{~b}$ and d) nearly overlap in the whole $\omega / t$ regime.

In Fig 5, we present the evolution of $A_{\mathbf{k}}(\omega)$ for $\mathbf{k}$ moving from $(\pi / 2, \pi / 2)$ towards $(0,0)$. All curves were computed using $N_{s t}=5213618$. Dark-shaded areas are graphic representations of $Z_{\mathbf{k}}$. The evolution of spectral functions with increasing values of $J / t$, calculated at $\mathbf{k}=(\pi / 2, \pi / 2)$, is presented in Fig.6. The two lowest string-like peaks are denoted with Roman numerals. Scaling as discussed in the beginning 


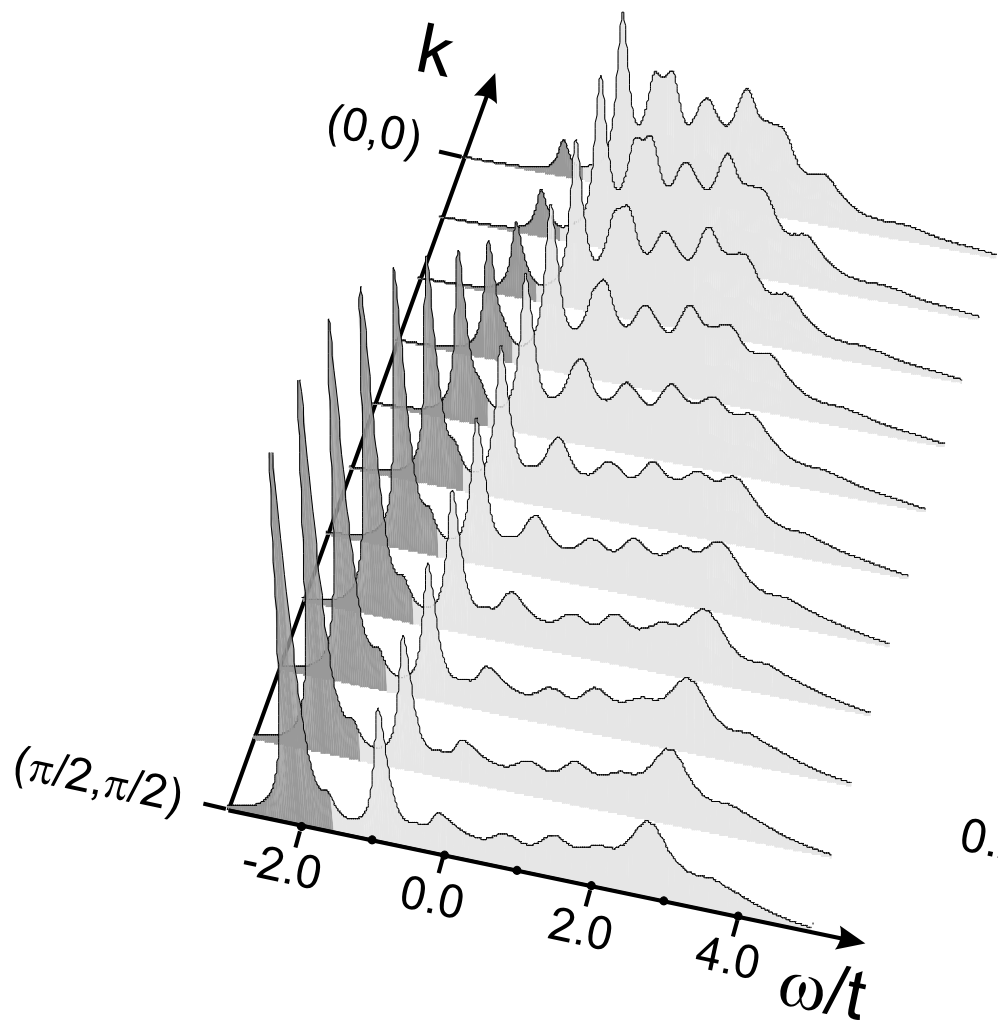

Figure 5: Spectral functions $A_{\mathbf{k}}(\omega)$ for wavevectors $\mathbf{k}=$ $(\pi / 2, \pi / 2)$ through $(0,0)$. In all cases we have used $J / t=0.3$, $N_{s t}=5213618$ and artificial damping $\epsilon=0.1$. Dark-shaded areas are proportional to the quasiparticle weight $Z_{\mathbf{k}}$.

of this section of the quasiparticle peak, and two lowest stringlike peak positions, $\omega_{0}, \omega_{1}$, and $\omega_{2}$, with $J / t$ can be qualitatively followed. With increasing $J / t$, the broad continuum at high $-\omega / t$ transforms into well defined peaks.

\section{CONCLUSIONS}

In conclusion, we have developed an efficient numerical approach for calculating physical properties of a doped AFM insulator in the zero-doping limit. The presented method is highly efficient, free of finite-size effects, and it allows for computation of physical properties at an arbitrary wavevector. EDLFS obviously has a few shortcomings: a) the method is limited to calculations in the zero-doping limit, b) due to the broken symmetry of the starting wavefunction, calculations are limited to the reduced AFM BZ, and c) results depend on the number of states $N_{s t}$ spanning the LFS. However, for most static as well as dynamic quantities convergence to the thermodynamic limit with increasing $N_{s t}$ can be achieved.

Using EDLFS, we have computed the quasiparticle energy, quasiparticle weight, and spectral functions and compared values to known and established analytical as well as numerical results. We found the best agreement with CE and SCBA calculations for the single-hole energy while for the quasipar-

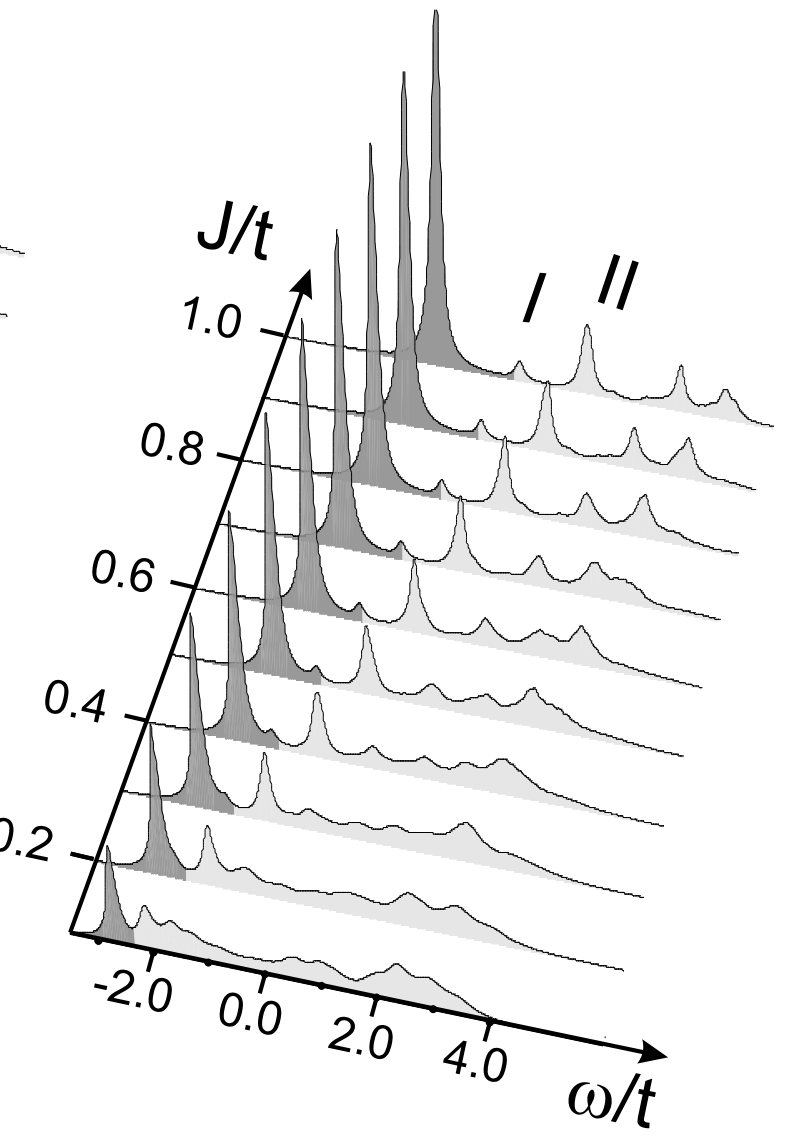

Figure 6: Spectral functions $A_{\mathbf{k}=(\pi / 2, \pi / 2)}(\omega)$ for various values of $J / t$ are shown. We have used $N_{s t}=5213618$ and artificial damping $\epsilon=0.1$. Dark-shaded areas are proportional to the quasiparticle weight $Z_{\mathbf{k}=(\pi / 2, \pi / 2)}$.

ticle weight at $J / t \lesssim 0.3$ best agreement was found with ED calculations obtained from the largest system of 32 sites. Our method with an already small number of LFS produces results for static quantities, such as the energy dispersion, the bandwidth and the quasiparticle weight, that are directly comparable to the state-of-the-art ED calculations on small lattices. Our simulations show that the quasiparticle weight around the band-minimum $\mathbf{k}=(\pi / 2, \pi / 2)$ remains finite in the thermodynamic limit. The quasiparticle peak is separated by a pseudo-gap from well defined string-like peaks. Comparing our results to ED calculations, we find a much smaller quasiparticle weight at the $\mathbf{k}=0$ point.

Our method can be easily extended to compute other static as well as dynamic quantities, e.g., various correlation functions in the vicinity of the doped hole and optical conductivity. Furthermore, it allows for the inclusion of additional higherorder terms in the Hamiltonian, such as the next-nearest neighbor hopping term that allows comparison of hole vs. electron doped AFM systems. The method can be easily extended to computation of bound two-hole properties by adding another hole to the LFS. EDLFS can also be adopted to computing single-hole properties of the $t-J$ model on the trian- 
gular lattice. In this case a different, $120^{\circ}$ ordered Neel state, should be used for the starting wavefunction, Ref. [29] Finally, by adopting the method of Ref. [30, 31], the present approach offers a natural extension to computation of the Holstein- $t-J$ model.

One of the authors (J.B.) acknowledges the warm hospitality during his visit at the Institute for Materials Research, Tohoku University, Sendai. J.B. also acknowledges stimulating discussions with A. Ramšak, I. Sega, and P. Prelovšek, M. Stout for providing editorial suggestions, J. Vidmar for encouragement, and the financial support of the Slovenian Research Agency under grant P1-0044. S.M. and T.T. acknowledge the financial support of the Next Generation Super Computing Project of Nanoscience Program, CREST, and Grantin-Aid for Scientific Research from MEXT.

[1] Y. Nagaoka, Phys. Rev. 147, 392 (1966).

[2] S. Sorella, Phys. Rev. B 53, 15119 (1996).

[3] A. Ramšak and P. Prelovšek, Phys. Rev. B 42, 10415 (1990).

[4] S. Schmitt-Rink, C. M. Varma, and A. E. Ruckenstein, Phys. Rev. Lett. 60, 2793 (1988).

[5] G. Martinez and P. Horsch, Phys. Rev. B 44, 317 (1991).

[6] Z. Liu and E. Manousakis, Phys. Rev. B 45, 2425 (1992).

[7] A. Ramšak and P. Horsch, Phys. Rev. B 57, 4308 (1998).

[8] W. F. Brinkman and T. M. Rice, Phys. Rev. B 2, 1324 (1970).

[9] S. A. Trugman, Phys. Rev. B 37, 1597 (1988).

[10] B. I. Shraiman and E. D. Siggia, Phys. Rev. Lett. 60, 740 (1988).

[11] P. Prelovšek, I. Sega, and J. Bonča, Phys. Rev. B 42, 10706 (1990).

[12] E. Dagotto, R. Joynt, A. Moreo, S. Bacci, and E. Gagliano, Phys. Rev. B 41, 9049 (1990).

[13] D. Poilblanc, T. Ziman, H. J. Schulz, and E. Dagotto, Phys. Rev.
B 47, 14267 (1993).

[14] P. W. Leung and R. J. Gooding, Phys. Rev. B 52, R15711 (1995).

[15] S. Sorella, Phys. Rev. B 46, 11670 (1992).

[16] A. S. Mishchenko, N. V. Prokofev, and B. V. Svistunov, Phys. Rev. B 64, 033101 (2001).

[17] A. S. Mishchenko and N. Nagaosa, Phys. Rev. Lett. 93, 036402 (2004).

[18] M. Brunner, F. F. Assaad, and A. Muramatsu, Phys. Rev. B 62, 15480 (2000).

[19] J. Inoue and S. Maekawa, J. Phys. Soc. Jpn. 59, 2110 (1990).

[20] E. Dagotto, Rev. Mod. Phys. 66, 763 (1994).

[21] A. Ramšak, P. Horsch, and P. Fulde, Phys. Rev. B 46, 14305 (1992).

[22] K. M. Shen, F. Ronning, D. H. Lu, W. S. Lee, N. J. C. Ingle, W. Meevasana, F. Baumberger, A. Damascelli, N. P. Armitage, L. L. Miller, et al., Phys. Rev. Lett. 93, 267002 (2004).

[23] O. Rösch, O. Gunnarsson, X. J. Zhou, T. Yoshida, T. Sasagawa, A. Fujimori, Z. Hussain, Z.-X. Shen, and S. Uchida, Phys. Rev. Lett. 95, 227002 (2005).

[24] T. Tohyama, Phys. Rev. B 70, 174517 (2004).

[25] D. N. Sheng, Y. C. Chen, and Z. Y. Weng, Phys. Rev. Lett. 77, 5102 (1996).

[26] S. E. Shawish and J. Bonča, Phys. Rev. B 74, 174420 (2006).

[27] K. J. von Szczepanski, P. Horsch, W. Stephan, and M. Ziegler, Phys. Rev. B 41, 2017 (1990).

[28] A. G. Malshukov and G. D. Mahan, Phys. Rev. Lett. 68, 2200 (1992).

[29] A. E. Trumper, C. J. Gazza, and L. O. Manuel, Physical Review B (Condensed Matter and Materials Physics) 69, 184407 (2004).

[30] J. Bonča, S. A. Trugman, and I. Batistić, Phys. Rev. B 60, 1633 (1999).

[31] J. Bonča, S. Maekawa, T. Tohyama, and P. Prelovšek, Work in progress (2007). 